\title{
Neutral oligosaccharide content of preterm human milk
}

\author{
Tarek Nakhla ${ }^{1}$, Daotian $\mathrm{Fu}^{2}$, David Zopf ${ }^{2}$, Nancy L. Brodsky ${ }^{1}$ and Hallam Hurt ${ }^{1}{ }^{*}$ \\ ${ }^{1}$ Division of Neonatology, Department of Pediatrics, Albert Einstein Medical Center, Philadelphia, PA, USA \\ ${ }^{2}$ Neose Technologies, Horsham, PA, USA
}

(Received 9 October 1998 - Revised 23 February 1999 - Accepted 8 June 1999)

\begin{abstract}
Human milk oligosaccharides are known to play a role in protection against certain infectious diseases. Previous reports indicate that the content of human milk oligosaccharides varies widely among individuals at term but such information on preterm milk is lacking. After removal of the fat, protein and most of the lactose from non-pooled human milk samples, a total neutral oligosaccharide fraction was isolated by ion-exchange chromatography followed by gel filtration. A Dionex high-performance anion-exchange chromatography system equipped with a pulsed electrometric detector was then employed to measure the levels of ten neutral oligosaccharides in the individual milk samples. Twenty-three milk samples from thirteen mothers who delivered at a mean gestational age of $29 \cdot 5$ (SD 3.1) weeks were collected between days 0 and 33 of lactation, and compared with three samples of term milk from two mothers. The ranges of the total and individual levels of the ten neutral oligosaccharides in preterm milk were similar to those in term milk. Further, as previously described in term milk, preterm milk exhibited a quantitative individual variation. This variation was independent of the gestational age, day of lactation, and postconceptional age. In conclusion, levels of ten neutral oligosaccharides did not differ between preterm and term human milk.
\end{abstract}

\section{Breast milk: Oligosaccharides: Lactation}

Breast-fed infants have fewer and less severe gastrointestinal and respiratory infections during the first year of life than formula-fed infants (Ogra \& Fishaut, 1990; Kunz \& Rudloff, 1993). The protective factors in human milk responsible for reduced infections have been the subject of decades of research. Besides the classical protective factors in human milk such as secretory immunoglobulin A, oligosaccharides, and lactoferrin, additional protective factors such as mucins, antioxidants, anti-inflammatory cytokines and leucocyte-altering components are being investigated (Buescher \& Malinowska, 1996).

The role of oligosaccharides as protective factors in human milk, while not fully defined, can be explained by at least two possible mechanisms. First, some oligosaccharides are growth factors for intestinal flora such as Bifidobacterium bifidus, which produce an intestinal environment unfavourable for many enteric pathogens (Ogra \& Fishaut, 1990; Kunz \& Rudloff, 1993; Miller et al. 1994). Second, oligosaccharides are potent inhibitors of bacterial adhesion to epithelial surfaces (Coppa et al. 1990; Kunz \& Rudloff, 1993; Pritchard \& Roth, 1995). Based on in vitro and animal studies, human milk oligosaccharides are suggested to prevent diarrhoeal diseases (Kunz \& Rudloff, 1993; Miller et al. 1994) otitis media
(Anderson et al. 1996), and urinary tract infections (Kunz \& Rudloff, 1993; Renner \& Sawatzki, 1993; Anderson et al. 1996).

In defatted human milk (composed of protein, lactose, and non-lactose carbohydrates), 85-90\% of the non-lactose carbohydrates are free oligosaccharides, with the remaining 10-15\% being protein-bound (Miller et al. 1994). Compared with milk from other species, human milk has moderate levels of oligosaccharides (Sabharwal et al. 1991; Kunz \& Rudloff, 1993; Renner \& Sawatzki, 1993; Miller et al. 1994). The high content of oligosaccharides in colostrum and mature milk for the term infant, and the gradual decrease of oligosaccharides over the first few months of lactation (Coppa et al. 1993) suggest that human milk is designed to protect the newborn at a time when the immune system is least mature. Indeed, there is an inverse correlation among mammals between maturity at birth and milk oligosaccharide levels (Pritchard \& Roth, 1995).

Investigators evaluating human milk oligosaccharides over the past five decades have established that the oligosaccharides present in human milk are structurally composed of six monosaccharide residues: glucose, galactose, $N$-acetylglucosamine, $N$-acetylgalactosamine, fucose and 
sialic acid (Kunz \& Rudloff, 1993; Miller et al. 1994). One simple classification of human milk oligosaccharides divides them into two categories, neutral oligosaccharides, which include fucosylated oligosaccharides, and acidic oligosaccharides, most of which contain sialic acid (Smith et al. 1978; Coppa et al. 1993). So far, more than 130 neutral and acidic oligosaccharides in human milk have been characterized, ranging in chain length from 3 to 11 (Kunz \& Rudloff, 1993; Renner \& Sawatzki, 1993). At least three genetically regulated systems, namely, the ABH system, the secretor gene, and the Lewis system, qualitatively affect the oligosaccharide content of the milk of a given mother (Ginsburg, 1972; Kobata, 1972; Issitt, 1985; Viverge et al. 1985, 1990a; Sabharwal et al. 1991; Walker, 1993). Quantitative individual variation also exists, making oligosaccharides one of the most variable components of human milk (Coppa et al. 1993; Kunz \& Rudloff, 1993; Miller et al. 1994). It is also known that the amount of oligosaccharides in term milk decreases throughout the lactation period (Carlson, 1985; Viverge et al. 1990b; Coppa et al. 1993; Renner \& Sawatzki, 1993; Miller et al. 1994), with a significant decrease starting from day 8 of lactation (Viverge et al. 1990b).

Although preterm infants, for a variety of reasons, are more vulnerable to infections than term infants there is little information about the oligosaccharide content of preterm milk. Because preterm milk is known to differ from term milk in regard to secretory immunoglobulin $\mathrm{A}, \mathrm{N}, \mathrm{Na}$, and $\mathrm{Cl}^{-}$content (Gross et al. 1980; Ogra \& Fishaut, 1990), we questioned whether preterm milk would have a higher content of oligosaccharides than term milk. Our specific aims were to evaluate the neutral oligosaccharide content of preterm milk and to compare our results from preterm milk with previously published data for term milk.

\section{Materials and methods}

\section{Preparation of samples}

Fifteen mothers whose babies were admitted to the Neonatal Intensive Care Unit at Albert Einstein Medical Center, Philadelphia, PA, USA, provided $5-10 \mathrm{ml}$ samples of human milk. Samples were collected by the mothers in sterile plastic bags, either by manual expression or using a breast pump, at different times of the day. Individual milk samples were immediately frozen at $-20^{\circ}$ until they were processed. We prepared the samples using a method similar to that of Stahl et al. (1994). Individual samples were thawed and centrifuged $\left(48400 \mathrm{~g}, 4^{\circ}, 20 \mathrm{~min}\right)$, and the supernatant layer of fat was removed. Addition of two volumes of ethanol $(950 \mathrm{ml} / \mathrm{l})$ to the defatted milk followed by overnight incubation at $4^{\circ}$ precipitated most of the lactose and protein. The sample was then centrifuged $\left(200 \mathrm{~g}, 4^{\circ}, 30 \mathrm{~min}\right)$ and the supernatant fraction (S1) transferred to another tube. The resulting pellet was resuspended in cold aqueous ethanol $(660 \mathrm{ml} / \mathrm{l})$, and centrifuged $(3000 \mathrm{~g}$, $\left.4^{\circ}, 15 \mathrm{~min}\right)$. The resulting pellet was discarded and the supernatant fraction (S2) collected. S1 and S2 were combined. The volume of $\mathrm{S} 1+\mathrm{S} 2$ was reduced to the original volume of the defatted sample by rotatory evaporation under vacuum and the remaining soluble protein was removed by ultrafiltration using Ultrafree-MC filters (10000 nominal molecular weight limit; Millipore Corporation, Bedford, MA, USA) centrifuged at $10000 \mathrm{~g}$ for $45-120 \mathrm{~min}$. The filtrate $(300-500 \mu \mathrm{l})$, containing both neutral and sialylated oligosaccharides, was subjected to ion-exchange chromatography on a $0.8 \mathrm{ml}$ Dowex AG $1 \times 2$ resin cartridge (acetate form, Bio-Rad Laboratories, Richmond, CA, USA). Neutral oligosaccharides were eluted with deionized water. Sialylated oligosaccharides were then eluted with $200 \mathrm{mM}$-sodium acetate and frozen for further analysis (not reported here). Neutral oligosaccharides were fractionated by size using a Bio-Gel P-4 gel filtration column $(580 \times 20 \mathrm{~mm}$; Bio-Rad Laboratories $)$, by elution with sodium azide $(0.2 \mathrm{~g} / \mathrm{l})$ at a flow rate of $0.2 \mathrm{ml} / \mathrm{min}$. Eight fractions, of $11 \mathrm{ml}$ each, were collected and stored at $-20^{\circ}$ until analysis.

\section{High-performance anion-exchange chromatography analysis of ten neutral oligosaccharides}

Lactose and ten oligosaccharide standards, 2'-fucosyllactose, 3-fucosyllactose, lacto- $N$-tetraose, lacto- $N$-neotetraose, lactodifucotetraose, lacto- $\mathrm{N}$-difucohexaose II, and lacto- $\mathrm{N}$ fucopentaoses (LNFP) I, II, III and V, were purchased from Oxford Glycosystems, Inc., Rosedale, NY, USA. Analysis of neutral oligosaccharides was performed on a Dionex Model DX500 high-performance anion-exchange chromatography system (Dionex, Sunnyvale, CA, USA) equipped with a GP40 gradient pump and an ED40 pulsed electrometric detector (HPAEC-PED). The system was controlled by, and data collected with, Dionex PeakNet software. Sample injections of $25 \mu l$ were made with a Thermo Separation Model AS3500 autosampler (Thermo Separation Products Inc., San Jose, CA, USA). The separation was carried out on a Dionex CarboPac PA1 HPAEC column $(4 \times 250 \mathrm{~mm})$. The separation programme began with a $7 \mathrm{~min}$ isocratic elution with $100 \mathrm{mM}-\mathrm{NaOH}$ at a flow rate of $1.0 \mathrm{ml} / \mathrm{min}$, followed by a $15 \mathrm{~min}$ linear gradient of 0-30 mM-sodium acetate in $100 \mathrm{~mm}-\mathrm{NaOH}$ (Thurl et al. 1996). Each oligosaccharide peak, identified by its retention time, was quantified based on the ratio of its area to that of a known amount $(10 \mu \mathrm{g} / \mathrm{ml})$ of its standard. The final concentration of each oligosaccharide in the defatted milk sample, expressed in $\mathrm{mg} / \mathrm{l}$, was calculated based on its total amount in all eight gel-filtration fractions and the original volume of the sample. All samples were prepared and analysed at Neose Technologies, Inc., Horsham, PA, USA.

\section{$H$ secretor and Lewis status of the mothers}

It is known that about $80 \%$ and $94 \%$ of individuals are $\mathrm{H}$ secretors and Lewis positive respectively (Issitt, 1985; Walker, 1993). Since some previous reports (Viverge et al. 1985, 1990a,b; Coppa et al. 1993; Thurl et al. 1996) included samples only from individuals of known $\mathrm{H}$ secretor or Lewis status, we identified the status of six of the fifteen mothers providing our samples.

Tubes containing individual saliva samples were placed in boiling water for $10 \mathrm{~min}$ and then centrifuged for $5 \mathrm{~min}$. The supernatant fractions were frozen until all samples were available (Issitt, 1985). The H secretor or Lewis status was 
Table 1. Characteristics of mothers and milk samples

\begin{tabular}{lccll}
\hline $\begin{array}{l}\text { Mother } \\
\text { no. }\end{array}$ & $\begin{array}{c}\text { Gestational } \\
\text { age (weeks) }\end{array}$ & $\begin{array}{c}\text { No. of } \\
\text { samples }\end{array}$ & $\begin{array}{c}\text { Day of } \\
\text { lactation }\end{array}$ & ABH secretor, Lewis status \\
\hline 1 & $27-28$ & 2 & 3,32 & H secretor, Lewis positive \\
2 & $31-32$ & 3 & $12,19,33$ & H secretor, Lewis positive \\
3 & 32 & 2 & 11,12 & H secretor, Lewis positive \\
4 & $29-30$ & 2 & 4,16 & H secretor, Lewis positive \\
5 & 27 & 1 & 23 & H secretor, Lewis positive \\
6 & $30-31$ & 2 & 4,25 & H secretor, Lewis negative \\
7 & $29-30$ & 2 & 8,19 & $\mathrm{~N} / \mathrm{A}$ \\
8 & 29 & 2 & 0,11 & $\mathrm{~N} / \mathrm{A}$ \\
9 & 32 & 2 & 4,22 & $\mathrm{~N} / \mathrm{A}$ \\
10 & $24-25$ & 2 & 6,31 & $\mathrm{~N} / \mathrm{A}$ \\
11 & $24-25$ & 1 & 3 & $\mathrm{~N} / \mathrm{A}$ \\
12 & $35-36$ & 1 & 24 & $\mathrm{~N} / \mathrm{A}$ \\
13 & $31-32$ & 1 & 4 & $\mathrm{~N} / \mathrm{A}$ \\
14 & 40 & 2 & 12,128 & $\mathrm{~N} / \mathrm{A}$ \\
15 & 40 & 1 & 4 & $\mathrm{~N} / \mathrm{A}$ \\
\hline
\end{tabular}

tested at the American Red Cross, Penn-Jersey Region, Reference Laboratory, Philadelphia, PA, USA. Le ${ }^{\mathrm{a}}$ and $\mathrm{Le}^{\mathrm{b}}$ erythrocyte typing was not done.

\section{Data analysis}

Gestational ages are summarized as means and standard deviations. Concentrations of oligosaccharides are summarized as medians and ranges. Relationships of oligosaccharide concentrations to gestational age, day of lactation, and postconceptional age were explored using Spearman correlations. Analyses were performed with SPSS for Windows, Version 6.1 (Statistical Package for the Social Sciences, Chicago, IL, USA).

\section{Ethical considerations}

This study was approved by the Institutional Review Board of Albert Einstein Medical Center. Written informed consent was obtained from the participating mothers.

Table 2. Concentrations $(\mathrm{mg} / \mathrm{l})$ of ten neutral oligosaccharides and their totals in twenty-three samples of preterm human milk from thirteen mothers

\begin{tabular}{|c|c|c|c|c|c|c|c|c|c|c|c|c|}
\hline \multirow{2}{*}{$\begin{array}{l}\text { Mother } \\
\text { no. }\end{array}$} & \multirow{2}{*}{$\begin{array}{l}\text { Day of } \\
\text { lactation }\end{array}$} & \multicolumn{11}{|c|}{ Oligosaccharides } \\
\hline & & $2^{\prime} \mathrm{FL}$ & $3 F L$ & LNT & LNnT & LDFT & LNFP I & LNFP II & LNFP III & LNFP V & LNDFH II & Totalł \\
\hline \multirow[t]{2}{*}{$1^{*}$} & 3 & 1491 & 414 & 304 & 120 & 164 & 562 & 104 & 101 & 18 & 44 & 3322 \\
\hline & 32 & 960 & 310 & 225 & 28 & 112 & 32 & 27 & 121 & 0 & 5 & 1820 \\
\hline \multirow[t]{3}{*}{$2^{*}$} & 12 & 1167 & 306 & 168 & 49 & 116 & 106 & 36 & 27 & 18 & 4 & 1997 \\
\hline & 19 & 1353 & 480 & 104 & 10 & 0 & 43 & 95 & 26 & 34 & 507 & 2652 \\
\hline & 33 & 1675 & 432 & 167 & 87 & 312 & 184 & 38 & 50 & 19 & 10 & 2973 \\
\hline \multirow[t]{2}{*}{$3^{*}$} & 11 & 1297 & 678 & 546 & 155 & 303 & 743 & 113 & 80 & 39 & 11 & 3965 \\
\hline & 12 & 1413 & 734 & 643 & 150 & 292 & 851 & 126 & 86 & 53 & 12 & 4360 \\
\hline \multirow[t]{2}{*}{$4^{*}$} & 4 & 344 & 503 & 304 & 152 & 185 & 82 & 147 & 37 & 11 & 13 & 1778 \\
\hline & 16 & 325 & 2635 & 1108 & 210 & 671 & 97 & 1338 & 194 & 232 & 280 & 7090 \\
\hline $5^{\star}$ & 23 & 394 & 591 & 148 & 10 & 227 & 32 & 93 & 27 & 0 & 69 & 1591 \\
\hline \multirow{2}{*}{$6+$} & 4 & 1130 & 0 & 113 & 105 & 23 & 373 & 0 & 0 & 8 & 2 & 1754 \\
\hline & 25 & 1239 & 11 & 130 & 123 & 60 & 303 & 0 & 5 & 13 & 1 & 1885 \\
\hline \multirow[t]{2}{*}{7} & 8 & 1303 & 133 & 187 & 81 & 139 & 250 & 16 & 40 & 0 & 1 & 2150 \\
\hline & 19 & 926 & 219 & 176 & 39 & 182 & 299 & 30 & 45 & 0 & 0 & 1916 \\
\hline \multirow[t]{2}{*}{8} & 0 & 1300 & 716 & 546 & 121 & 446 & 526 & 115 & 93 & 57 & 14 & 3934 \\
\hline & 11 & 1134 & 516 & 308 & 62 & 270 & 234 & 46 & 60 & 28 & 0 & 2658 \\
\hline \multirow[t]{2}{*}{9} & 4 & 1060 & 41 & 994 & 152 & 110 & 887 & 0 & 122 & 66 & 6 & 3438 \\
\hline & 22 & 539 & 57 & 977 & 50 & 43 & 755 & 0 & 92 & 16 & 1 & 2530 \\
\hline \multirow[t]{2}{*}{10} & 6 & 1092 & 108 & 124 & 64 & 166 & 207 & 66 & 10 & 0 & 1 & 1838 \\
\hline & 31 & 1195 & 880 & 519 & 140 & 396 & 1181 & 118 & 129 & 44 & 6 & 4608 \\
\hline 11 & 3 & 0 & 583 & 248 & 59 & 0 & 5 & 129 & 65 & 17 & 668 & 1774 \\
\hline 12 & 24 & 343 & 182 & 159 & 22 & 64 & 25 & 23 & 12 & 0 & 7 & 837 \\
\hline & 4 & 1634 & 488 & 54 & 43 & 1269 & 205 & 48 & 125 & 15 & 15 & 3896 \\
\hline Ranges & $0-33$ & $0-1675$ & $0-2635$ & 54-1108 & $10-210$ & $0-1269$ & $5-1181$ & $0-1338$ & $0-194$ & $0-232$ & $0-668$ & $837-7090$ \\
\hline
\end{tabular}

2'FL, 2' -fucosyllactose; 3FL, 3-fucosyllactose; LNT, lacto- $N$-tetraose; LNnT, lacto- $N$-neotetraose; LDFT, lactodifucotetraose; LNFP, lacto- $N$-fucopentaose; LNDFH II, lacto- $N$-difucohexaose II.

${ }^{*}$ Lewis positive.

$\dagger$ Lewis negative.

$\ddagger$ Sum of the ten neutral oligosaccharides. 
(a)

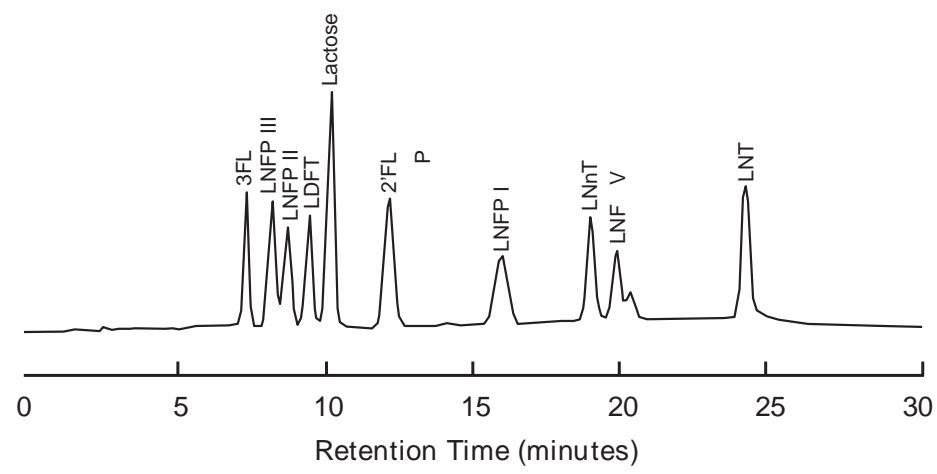

(b)
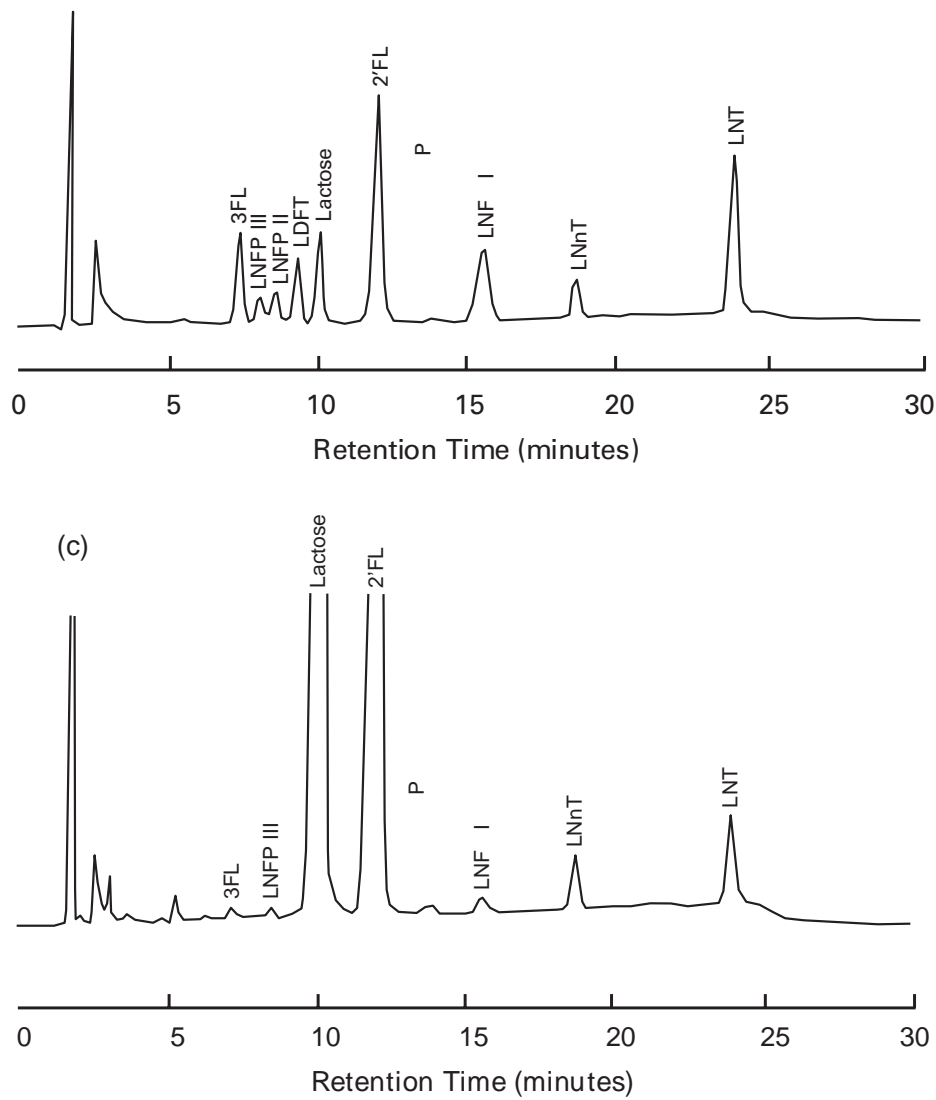

Fig. 1. Three chromatograms from high-performance anion-exchange chromatography with pulsed electrometric detection (PED) of ten neutral oligosaccharides (N-OS) identified by their retention times. (a) Lactose and nine of the $\mathrm{N}$-OS standards (10 $\mu \mathrm{g} / \mathrm{ml})$. The nine $\mathrm{N}$-OS peaks are: 3-fucosyllactose (3FL), lacto- $N$-fucopentaose III (LNFP III), lacto- $N$-fucopentaose II (LNFP II), lactodifucotetraose (LDFT), 2'-fucosyllactose (2'FL), lacto- $N$-fucopentaose I (LNFP I), lacto- $N$-neotetraose (LNnT), lacto- $N$-fucopentaose V (LNFP V), and lacto- $N$-tetraose (LNT). LNDFH II elutes at $5.9 \mathrm{~min}$ (not shown). (b) N-OS recovered in a fraction of preterm milk from a Lewis positive mother (no. 3). (c) N-OS recovered in a fraction of preterm milk from a Lewis negative mother (no. 6). Note the different scale of the $Y$ axis. 


\section{Results}

Twenty-three samples from thirteen mothers who delivered preterm infants at a mean gestational age of 29.5 (SD 3.1) weeks, were collected between days 0 and 33 of lactation. Three samples were collected from two mothers who delivered at term. All six mothers tested for $\mathrm{H}$ secretor and Lewis status were $\mathrm{H}$ secretors, five were Lewis positive, and one was Lewis negative (Table 1).

Table 2 presents the concentrations of the ten neutral oligosaccharides and their totals in the twenty-three samples of preterm milk. The wide ranges of the concentrations of individual oligosaccharides and their totals indicate a considerable variation among individuals. In the individual identified as Lewis negative (mother no. 6), no LNFP II was detected in any of the gel filtration fractions, an observation consistent with previously reported qualitative variability dependent on the mother's heritable Lewis status (Issitt, 1985; Walker, 1993).

For nine mothers of preterm infants, a second or third milk sample was collected within a period ranging from 11 to $29 \mathrm{~d}$ after the first sample. In four mothers (nos. 1, 7, 8, 9) there was an 11-45\% decrease in total neutral oligosaccharide levels, whereas in five mothers (nos. 2, 3, 4, 6, 10) there was an 8-300\% increase in total neutral oligosaccharide levels (Table 2). There was no correlation between oligosaccharide levels (individual or total) and gestational age, or postconceptional age (all $P \geq 0 \cdot 21$, results not shown). While there was little variation with day of lactation, the range of collection was only $33 \mathrm{~d}$, therefore a more detailed study is needed to assess variation with duration of lactation.

Fig. 1(a) shows an HPAEC-PED chromatogram of the mixture of lactose and nine of the ten neutral oligosaccharide standards. Fig. 1(b) shows a representative chromatogram in which eight neutral oligosaccharides recovered in preterm milk from mother no. 3 (Lewis positive) were detected. It should be noted that not all oligosaccharides present in a milk sample were detected in all eight fractions analysed after gel filtration. For example, LNFP V which was detected in both samples from mother no. 3 (Table 2) was not present in the fraction chosen for illustration in Fig. 1(b). Fig. 1(c) shows a representative chromatogram of one of the eight gel filtration fractions of a sample from mother no. 6 (Lewis negative). As noted earlier, no LNFP II was detected in any of the gel filtration fractions from this donor (Table 2).

Table 3 shows the median concentrations and ranges of the ten neutral oligosaccharides and their total in the twentythree preterm milk samples from thirteen mothers (a summary of the data in Table 2) and three term samples from two mothers in the present study. As mentioned earlier, individual oligosaccharides and their total show individual variation as indicated by the wide ranges in their concentrations in both preterm and term milk samples. Table 3 also presents previously reported concentrations in pooled term milk (Kunz \& Rudloff, 1993) as well as concentrations from one non-pooled term milk sample (Thurl et al. 1996). By inspection, the ranges and the median concentrations of the ten neutral oligosaccharides and their totals in preterm milk were not substantially different from those from either our term milk or previously reported data.

Table 4 shows the median concentrations and ranges of the ten neutral oligosaccharides and their totals in ten preterm milk samples from five Lewis-positive mothers, as well as data from the one term milk sample from a Lewispositive mother reported by Thurl et al. (1996). Individual oligosaccharides and their totals continue to exhibit wide variations despite being only from Lewis positive individuals. By inspection, preterm milk levels were similar to those in term milk. The wide individual variations, our sample size, as well as the limited number of samples in previous reports, precluded statistical comparison.

\section{Discussion}

Oligosaccharides in human milk play a role in the development of specific intestinal flora in breast-fed infants (Ogra \& Fishaut, 1990; Kunz \& Rudloff, 1993). In addition,

Table 3. Median concentrations and ranges (mg/l) of ten neutral oligosaccharides in breast-milk samples from fifteen mothers compared with previously reported values

\begin{tabular}{|c|c|c|c|c|c|c|}
\hline \multirow{3}{*}{$\begin{array}{l}\text { Neutral } \\
\text { oligosaccharide }\end{array}$} & \multicolumn{4}{|c|}{ Present study } & \multirow{2}{*}{\multicolumn{2}{|c|}{ Previous reports }} \\
\hline & \multicolumn{2}{|c|}{ Preterm milk ( $n$ 23) } & \multicolumn{2}{|c|}{ Term milk ( $n$ 3) } & & \\
\hline & Median & Ranan & Modian & Ranan & $\begin{array}{l}\text { Pooled term } \\
\text { milk }^{*}\end{array}$ & $\begin{array}{l}\text { Term milk } \\
\quad(n 1)\end{array}$ \\
\hline 2'FL & 1134 & $0-1675$ & 1273 & $1152-1316$ & N/A & 1840 \\
\hline $3 F L$ & 432 & $0-2635$ & 159 & $79-396$ & $\mathrm{~N} / \mathrm{A}$ & 460 \\
\hline LNT & 225 & 54-1108 & 169 & $167-293$ & $500-1500$ & 860 \\
\hline LNnT & 81 & $10-210$ & 48 & $42-122$ & $\mathrm{~N} / \mathrm{A}$ & 110 \\
\hline LDFT & 166 & $0-1269$ & 84 & $0-175$ & N/A & 170 \\
\hline LNFP I & 234 & $5-1181$ & 285 & $153-492$ & $1000-1500$ & 670 \\
\hline LNFP II & 48 & $0-1338$ & 0 & $0-86$ & $500-1000$ & 200 \\
\hline LNFP III & 60 & $0-194$ & 63 & $27-135$ & $\mathrm{~N} / \mathrm{A}$ & 280 \\
\hline LNFP V & 17 & $0-232$ & 47 & $4-70$ & $\mathrm{~N} / \mathrm{A}$ & $\mathrm{N} / \mathrm{A}$ \\
\hline LNDFH II & 7 & $0-668$ & 58 & 5-631 & N/A & 250 \\
\hline Total & 2530 & $837-7090$ & 2432 & $2022-3077$ & $3000-6000$ & 4480 \\
\hline
\end{tabular}

2'FL, 2'-fucosyllactose; 3FL, 3-fucosyllactose; LNT, lacto- $N$-tetraose; LNnT, lacto- $N$-neotetraose; LDFT, lactodifucotetraose; LNFP, lacto- $N$-fucopentaose; LNDFH II, lacto- $N$-difucohexaose II; N/A, not analysed.

*Values from Kunz \& Rudloff (1993).

† Values from Thurl et al. (1996). 
Table 4. Median concentrations and ranges $(\mathrm{mg} / \mathrm{l})$ of ten neutral oligosaccharides in ten preterm human milk samples from five Lewis positive mothers compared with previously reported data on one Lewis positive sample

\begin{tabular}{lccc}
\hline & \multicolumn{3}{c}{ Lewis positive mothers } \\
\cline { 2 - 3 } Neutral & \multicolumn{2}{c}{ Preterm milk $(n 10)$} & \\
\cline { 2 - 3 } oligosaccharide & Median & Range & Term milk* $(n$ 1) \\
\hline 2'FL & 1232 & $325-1675$ & 1840 \\
3FL & 491 & $306-2635$ & 460 \\
LNT & 264 & $104-1108$ & 860 \\
LNnT & 103 & $10-210$ & 110 \\
LDFT & 206 & $0-671$ & 170 \\
LNFP I & 101 & $32-851$ & 670 \\
LNFP II & 99 & $27-1338$ & 200 \\
LNFP III & 65 & $26-194$ & 280 \\
LNFP V & 18 & $0-232$ & $\mathrm{~N} / \mathrm{A}$ \\
LNDFH II & 12 & $4-507$ & 250 \\
Total & 2813 & $1591-7090$ & 4840 \\
\hline
\end{tabular}

2'FL, 2'-fucosyllactose; 3FL, 3-fucosyllactose; LNT, lacto- $N$-tetraose; LNnT lacto- $N$-neotetraose; LDFT, lactodifucotetraose; LNFP, lacto- $N$-fucopentaose; LNDFH, lacto- $N$-difucohexaose II; N/A, not analysed.

*Values from Thurl et al. (1996).

oligosaccharides as well as glycoproteins are considered to be potent inhibitors of pathogen adhesion to epithelial surfaces (Ashknazi \& Mirelman, 1987; Coppa et al, 1990; Kunz \& Rudloff, 1993; Renner \& Sawatzkij, 1993; Zopf \& Roth, 1996). In most instances, the initiating event of an infection is the attachment of the pathogen (bacterium, virus, protozoan, or fungus) to a target cell in the host. Attachment occurs when protein molecules (lectins or adhesins) on the pathogen form non-covalent complexes with specific carbohydrates on the surface of a target cell. Oligosaccharides found in mucous layers interact with adhesins on pathogens. This interaction blocks the attachment of pathogens to host target cells, thus preventing the infection resulting from such an attachment (Ginsburg, 1972; Pritchard \& Roth, 1995; Zopf \& Roth, 1996).

Several oligosaccharides naturally occurring in human milk are known to act as receptors for microbial organisms: fucosylated oligosaccharides are known receptors for Escherichia coli, lacto- $N$-tetraose and lacto- $N$-neotetraose are receptors for Streptococcus pneumoniae, and several sialylated oligosaccharides are considered to be receptors for Helicobacter pylori, Mycoplasma pneumoniae and influenza viruses A, B, and C (Kunz \& Rudloff, 1993).

Investigations of human milk suggest that many protective factors, including oligosaccharides, are responsible for the reduced risk of infections in breast-fed infants. Most reports, however, have evaluated only term milk oligosaccharides with few reports on the oligosaccharide content of preterm milk. Kunz \& Rudloff (1993) suggest that compositional changes of oligosaccharides in preterm milk occur during lactation in the same manner as in term milk, with the highest amounts of oligosaccharides being found at early stages of lactation. Miller et al. (1994) reported levels of monosaccharides (hydrolysed oligosaccharides) in preterm milk from two mothers but did not evaluate individual oligosaccharides.

To our knowledge, this is the first report of individual levels of neutral oligosaccharides in a large number of nonpooled samples of preterm milk, at very early gestational ages, including ten samples from five known Lewis-positive mothers. Previously published studies of human milk oligosaccharides used a variety of analytical methods. Some investigators evaluated only individual monosaccharides (Carlson, 1985; Viverge et al. 1990a,b; Miller et al. 1994). Coppa et al. (1993) prepared their own standards of a mixture of oligosaccharides by isolating oligosaccharides from pooled human milk from mothers of the same Lewis phenotype, then using HPLC to identify and quantify one peak for the mixture of oligosaccharides. Viverge et al. (1990a) used gel chromatography followed by paper chromatography to identify nine oligosaccharides (five neutral and four sialylated) in term milk from a Lewis positive donor. Kunz et al. (1993) and Thurl et al. (1996) used high-pH anion-exchange chromatography with pulsed amperometric detection to identify and quantify individual oligosaccharides. Some investigators analysed pooled term milk from different individuals (Kunz \& Rudloff, 1993), while others analysed individual samples (Carlson, 1985; Viverge et al. 1990a,b; Coppa et al. 1993; Miller et al. 1994). We removed the protein and most of the lactose from the samples, and used gel filtration, followed by HPAEC$\mathrm{PED}$, to identify and quantitate individual neutral oligosaccharides based on external standards. We did not use pooled milk but analysed the samples individually. We compared our results with those of Kunz et al. (1993) and Thurl et al. (1996) due to the similarity between our methods and theirs. However, as mentioned earlier, the wide individual variations in the ranges of oligosaccharides and the lack of a large number of samples in our study and other reported studies precluded statistical comparison.

In summary, we report that levels of ten neutral oligosaccharides in twenty-three individual samples of preterm human milk did not differ substantially from those of term milk. In addition to known variation in oligosaccharide composition related to the mother's Lewis phenotype, oligosaccharide levels varied over a wide range, regardless of gestational age, day of lactation, or postconceptional age. Defining the oligosaccharide levels and their temporal changes, if any, in preterm milk is a step toward improved understanding of the immunological needs of the preterm infant.

\section{References}

Anderson B, Porras O, Hanson LÅ, Lagergård T \& Edén CS (1996) Inhibition of attachment of Streptococcus pneumoniae and Haemophilus influenzae by human milk and receptor oligosaccharides. Journal of Infectious Diseases 153, 232-237.

Ashknazi S \& Mirelman D (1987) Nonimmunoglobulin fraction of human milk inhibits the adherence to certain enterotoxigenic Escherichia coli strains to guinea pig intestinal tract. Pediatric Research 22, 130-134.

Buescher ES \& Malinowska I (1996) Soluble receptors and cytokine antagonists in human milk. Pediatric Research 40, 839-844.

Carlson S (1985) N-Acetylneuraminic acid concentrations in human milk oligosaccharides and glycoproteins during lactation. American Journal of Clinical Nutrition 41, 720-726. 
Coppa GV, Gabrielli O, Giorgi P, Catassi C, Montanari M, Varaldo P \& Nichols B (1990) Preliminary study of breast feeding and bacterial adhesion to uroepithelial cells. Lancet 335, 569-571.

Coppa GV, Gabrielli O, Pierani P, Catassi C, Carlucci A \& Giorgi PL (1993) Changes in carbohydrate composition in human milk over 4 months of lactation. Pediatrics 91, 637-641.

Ginsburg V (1972) Enzymatic basis for blood groups in man. Advances in Enzymology 36, 131-149.

Gross SJ, David RJ, Bauman L \& Tomarelli RM (1980) Nutritional composition of milk produced by mothers delivering preterm. Journal of Pediatrics 96, 641-644.

Issitt PD (1985) Applied Blood Group Serology, 3rd ed., pp. 63-66 and 170-188. Miami, FL: Montgomery Scientific Publications.

Kobata A (1972) Isolation of oligosaccharides from human milk. Methods in Enzymology 28, 262-271.

Kunz C \& Rudloff S (1993) Biological functions of oligosaccharides in human milk. Acta Paediatrica 82, 903-912.

Miller JB, Bull S, Miller J \& McVeagh P (1994) The oligosaccharide composition of human milk: temporal and individual variations in monosaccharide components. Journal of Pediatric Gastroenterology and Nutrition 19, 371-376.

Ogra PL \& Fishaut M (1990) Human breast milk. In Infectious Diseases of the Fetus and Newborn Infant, 3rd ed., pp. 68-88 [JS Remington and JO Klein, editors]. Philadelphia, PA: WB Saunders Company.

Pritchard D \& Roth S (1995) Enzymatic production of complex carbohydrates: a new approach to medical applications of heterooligosaccharides. SIM News 45, 214-220.

Renner B \& Sawatzki G (1993) New Perspectives in Infant Nutrition Symposium, Antwerp 1992, pp.3-31 and 43-49. New York, NY: Thieme Medical Publishers, Inc.
Sabharwal H, Sjöblad S \& Lundblad A (1991) Affinity chromatographic identification and quantitation of blood group A-active oligosaccharides in human milk and feces of breast-fed infants. Journal of Pediatric Gastroenterology and Nutrition 12, 474-479.

Smith DF, Zopf DA \& Ginsburg V (1978) Sialyl oligosaccharides from milk. Methods in Enzymology 50, 221-229.

Stahl B, Thurl S, Zeng J, Karas M, Hillenkamp F, Steup M \& Sawatzki G (1994) Oligosaccharides from human milk as revealed by matrix-assisted laser desorption/ionization mass spectrometry. Annals of Biochemistry 223, 218-226.

Thurl S, Werner BM \& Sawatzki G (1996) Quantification of individual oligosaccharide compounds from human milk using high-pH anion exchange chromatography. Annals of Biochemistry 235, 202-206.

Viverge D, Grimmonprez L, Cassanas G, Bardet L, Bonnet H \& Solere M (1985) Variations of lactose and oligosaccharides in milk from women of blood types secretor A or $\mathrm{H}$, secretor Lewis, and secretor $\mathrm{H} /$ nonsecretor Lewis during the course of lactation. Annals of Nutrition and Metabolism 29, 1-11.

Viverge D, Grimmonprez L, Cassanas G, Bardet L \& Solere M (1990a) Variations in oligosaccharides and lactose in human milk during the first week of lactation. Journal of Pediatric Gastroenterology and Nutrition 11, 361-364.

Viverge D, Grimmonprez L, Cassanas G, Bardet L \& Solere M $(1990 b)$ Discriminant carbohydrate components of human milk according to donor secretor types. Journal of Pediatric Gastroenterology and Nutrition 11, 365-370.

Walker RH (1993) ABO, H and P blood groups and structurally related antigens. In Technical Manual, 11th ed., pp. 203-228. Bethesda, MD: American Association of Blood Banks.

Zopf D \& Roth S (1996) Oligosaccharide anti-infective agents. Lancet 347, 1017-1021. 